\title{
Scanning electron microscopy methods for analysis of polymer nanocomposites
}

\author{
D. A. Dikin, K. M. Kohlhaas, G. H. B. Dommett, S. Stankovich, R. S. Ruoff \\ Department of Mechanical Engineering, Northwestern University, 2145 Sheridan Road, Evanston, \\ IL 60208
}

The properties associated with polymer-matrix composites are a function of the filler size, shape, dispersion, and of the matrix-filler interaction. The morphological and structural analysis of nanocomposites is often done with transmission electron microscopy (TEM), where extensive imaging is required to ensure a representative view of the material. Carbon-based nanofillers, such as carbon nanotubes, thin graphitic platelets, carbon black, etc., also have very low TEM contrast when embedded in a polymer matrix. By combining several scanning electron microscopy (SEM) techniques detailed information of the nanocomposite morphology, conductivity, and even of modes of fracture, can be obtained at the nanometer scale. We have made and tested a series of nanocomposite samples with different carbon fillers and concentrations. SEM image data of such samples has been correlated to direct current electrical measurements and tensile loading tests.

SEM resolution is traditionally associated with the electron beam spot size, which is smaller at higher acceleration voltage (AV). For high resolution microscopy of nanostructures, however, resolution and information-passing capacity [1] are inherently linked to the sample volume where secondary electrons are created and from which they are collected, and also to the efficiency of the low energy electron detectors. At low AVs, primarily surface features are seen, as illustrated in Fig. 1, which also shows that carbon nanotube contrast has a different sign at different AVs. Their contrast/brightness values are also a function of the electron flux per unit time, and discharge mechanisms, as has been discussed for the charge contrast imaging technique [2].

The low AV imaging can be compared to the combination of topography imaging by atomic force microscopy and scanning Kelvin probe microscopy. The sub-surface imaging technique [3] is however more valuable for the goal of testing the conductivity of nanocomposites and of the analyzing the distribution and structure of the filler particles. Fig. 2 shows a comparison between surface and sub-surface imaging. The remarkable difference between low and high AV images is because the iso-conductance surfaces do not coincide with the physical surface of the sample probed by low energy primary electrons. Contrast in sub-surface imaging is primarily associated with the electrical conductivity of the embedded particles, diffusion of the electrical charges into the insulating matrix, and the existence of a percolating network that connects particles into isolated or "infinite" clusters. The depth of sub-surface imaging is a function of filler concentration, distribution, network conductivity, and also to some extent, the energy of the primary electrons; thus in principle, all of these parameters can be analyzed. However, two limiting cases can not be avoided - only surface imaging is possible for insulating composites, and also for heavily packed and highly electrically conductive composites.

These limitations for bulk samples can however be circumvented by preparing (microtomed) thin sample slices and placing them onto a moderately conductive substrate, for example a silicon wafer with a thin native oxide surface. Such a substrate for the thin slice creates an "electrostatic mirror" for electrons, which can readily transmit via the conductive particles through the insulating matrix 
and efficiently be emitted out of the sample surface. In this approach, individual particles or their clusters can be visualized, so that working is not restricted to only the vicinity of the percolation transition in the nanocomposites.

Our methodology and work demonstrates that SEM imaging can offer a versatile and rapid analysis of conductive polymer nanocomposites for a wide range of filler concentrations.

References

[1] J. Orloff (eds.), Handbook of Charged Particle Optics, CRC, New York, 1997.

[2] F. Ludek et al., Scanning, 23 (2001) 36.

[3] J. Loos, et al., Ultramicroscopy, 104 (2005) 160.

[4] This research was supported by the NASA University Research, Engineering and Technology Institute on Bio Inspired Materials (BIMat) under award No. NCC-1-02037, and by the National Science Foundation (NIRT Program, Grant No. 0304506, Dr. Ken P. Chong, Program Director)
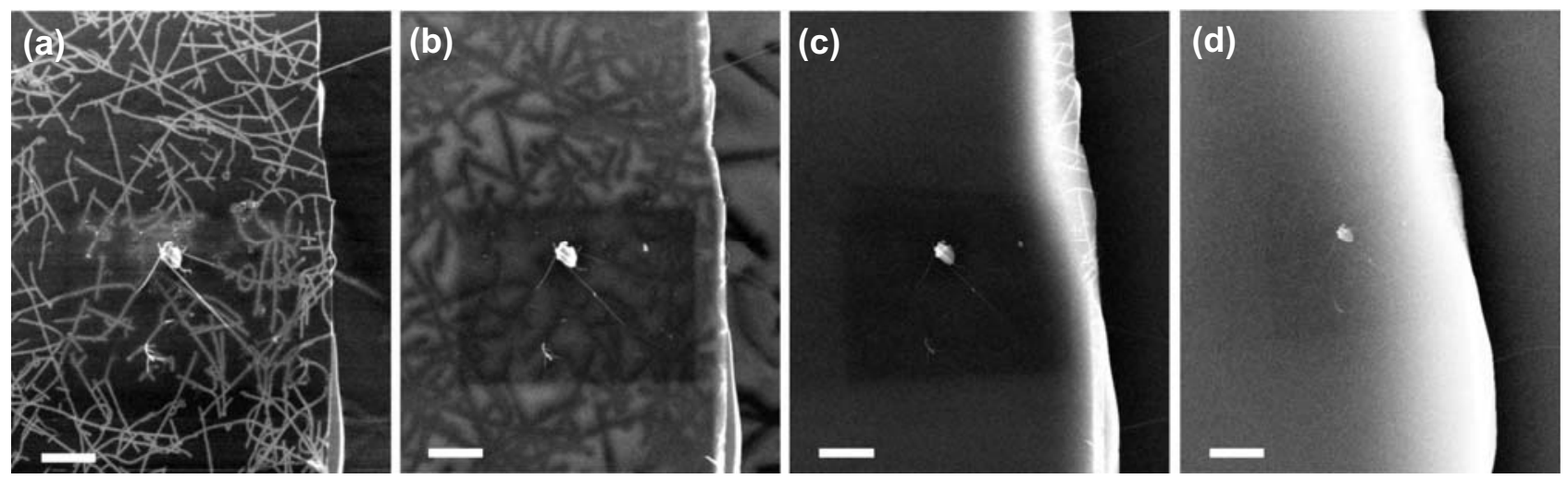

FIG. 1. SEM secondary electron images of carbon nanotubes dispersed on a Si substrate obtained at (a) $0.6 \mathrm{kV}$, (b) $2.5 \mathrm{kV}$, (c) $10 \mathrm{kV}$, and (d) $20 \mathrm{kV}$ acceleration voltages. Scale bar is $1 \mu \mathrm{m}$.
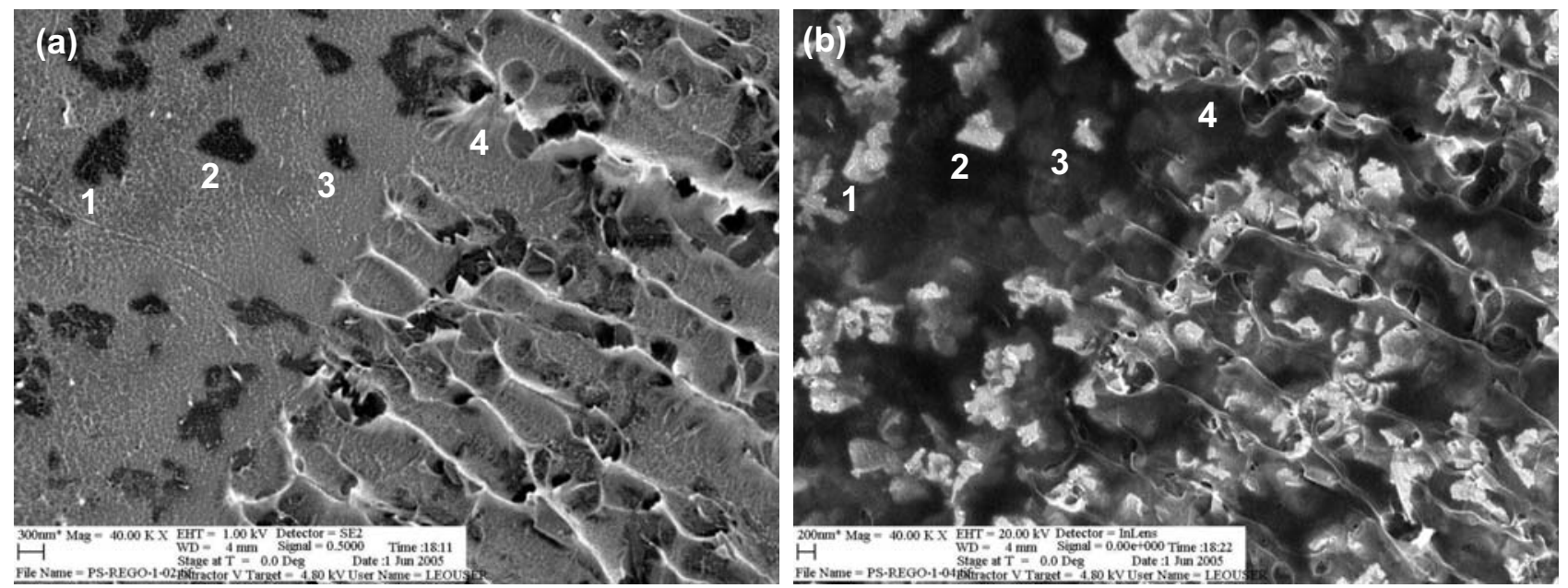

FIG. 2. SEM micrograph of a polymer-graphite nanoparticle composite taken from the same sample area at two different incident beam energies. Features of interest are marked by the same numbers on both images. (a) At $1 \mathrm{kV}$ : surface-type imaging. (b) At $20 \mathrm{kV}$ : subsurface imaging. 